\title{
Multicomponent Polysaccharide Essential Formula of Wound Healing Medicines Enriched with Fibroblast Growth Factor
}

\author{
Ekaterina V. Silina, $\mathrm{PhD}, \mathrm{ScD}^{1 *}$; Nikolay V. Khokhlov, $\mathrm{PhD}, \mathrm{ScD}^{1}$; Victor A. Stupin, $\mathrm{PhD}$, \\ $\mathrm{ScD}^{2}$; Natalya E. Manturova, $\mathrm{PhD}, \mathrm{ScD}^{2}$; Vitaliy I. Vasin ${ }^{2}$; Evgeniy V. Velikanov, $\mathrm{PhD}^{3}$; \\ Anton L. Popov, $\mathrm{PhD}^{4}$; Vadim B. Gavrilyuk, $\mathrm{PhD}, \mathrm{ScD}^{4}$; Elena B. Artyushkova, $\mathrm{PhD}, \mathrm{ScD}^{5}$; \\ Mikhail P. Gladchenko, $\mathrm{PhD}^{5}$; Alexander V. Ivanov, $\mathrm{PhD}, \mathrm{ScD}^{5}$; Victor T. Dudka, $\mathrm{PhD}$, \\ $\mathrm{ScD}^{5}$; Alexey A. Kryukov, $\mathrm{PhD}^{5}$; Yuri V. Furman, $\mathrm{PhD}, \mathrm{ScD}^{5}$; Anton N. Kaplin, $\mathrm{PhD}, \mathrm{ScD}^{5}$; \\ Alexander V. Anikanov; ; Marina A. Chernyatina, $\mathrm{PhD}, \mathrm{ScD}^{5}$; Ekaterina V. Anurova, $\mathrm{PhD}, \mathrm{ScD}^{5}$ \\ II.M. Sechenov First Moscow State Medical University, Moscow, Russia \\ ${ }_{2}^{2}$ Pirogov Russian National Research Medical University (RNRMU), Moscow, Russia \\ ${ }^{3}$ Moscow Regional Research Clinical Institute named after M.F. Vladimirsky, Moscow, Russia \\ ${ }^{4}$ Institute of Theoretical and Experimental Biophysics RAS, Pushchino, Russia \\ ${ }^{5}$ Kursk State Medical University, Kursk, Russia
}

\begin{abstract}
The purpose of this research work was the creation of a safe and effective wound healing drug, in the multicomponent polysaccharide base of which the fibroblast growth factor is integrated in the optimal concentration.

Materials and Methods: The present work was carried out in three stages. In Stage 1, the essential formula of a wound healing drug was created. In order to optimize the composition of the base formula, studies were conducted with various concentrations of structure-forming polysaccharides. In Stage 2, we performed technical tests of the gel composition of the medical product on hMSCs culture, and determined the optimal concentration of the growth factor, which was added to the base formula developed at Stage 1 in different concentrations. In Stage 3, an experimental safety study of the developed gel composition, including growth factors, was performed on laboratory animals. In an experimental safety study (subchronic toxicity) on a wound model, sexually mature male rats and Wistar females of the same age (8-9 months) were used. In all animals, 2 wounds of standard size were modeled on the back. Taking into account the weight and gender, rats were distributed evenly into two experimental groups. Group 1 included rats we treated with a polysaccharide gel based on polymers. This polysaccharide gel included rhFGF-b. Group 2 was used as control (without treatment).

Results: The laboratory, histological and gravimetric studies of the internal organs and wounds of the animals in the experimental and control groups did not reveal any regular pathomorphological changes indicating the presence of the general toxicological properties of the studied products. This indicates the safety in vivo of the product being developed.

Conclusion: The studies conducted in vitro and in vivo allow us to proceed with studies determining the effectiveness of the developed wound healing medical product on laboratory animals. (International Journal of Biomedicine. 2019;9(3):247-250.)
\end{abstract}

Key Words: regeneration $\bullet$ wound healing $\bullet$ fibroblast growth factor $\bullet$ stem cells

\section{Introduction}

The problem of wound healing in any medical specialty related to surgery is always important. Given the increasing

*Corresponding author: Prof. Ekaterina V. Silina, PhD, ScD. Professor of the Department of Human Pathology of the I.M. Sechenov First Moscow State Medical University. Moscow, Russia; E-mail: silinaekaterina@mail.ru prevalence in the world population of cardiovascular diseases, diabetes mellitus, obesity, and metabolic syndrome, the hard-to-cure chronic wounds even more worsen the global problem, which is associated with significant economic costs. ${ }^{(1-3)}$ Therefore, to provide healing of acute and chronic wounds, ever new medicines and medical devices are constantly being developed that use biological mechanisms of cell regeneration, ${ }^{(4-9)}$ including growth factors and stem cells, which offer high hopes. ${ }^{(10-14)}$ However, despite the current 
successes, the task of developing tools for fast and high-quality epithelization of acute and chronic wounds remains vital.

The purpose of this research work was the creation of a safe and effective wound healing drug, in the multicomponent polysaccharide base of which the fibroblast growth factor is integrated in the optimal concentration.

\section{Materials and Methods}

The present work was carried out in three stages. In Stage 1, the essential formula of a wound healing drug was created. In order to optimize the composition of the base formula, studies were conducted with various concentrations of structure-forming polysaccharides. The concentration of pectin (Sigma Aldrich) was selected in the range of 0 to $5 \mathrm{~g} / \mathrm{l}$ with an increment of $0.5 \mathrm{~g} / \mathrm{l}$. The concentration of the substance alginate (Sigma Aldrich) was selected in the range of 0 to $1 \mathrm{~g} / 1$ with an increment of $0.1 \mathrm{~g} / \mathrm{l}$. The concentration of the substance chitosan (Sigma Aldrich) was selected in the range of 0 to $1 \mathrm{~g} / 1$ with an increment of $0.1 \mathrm{~g} / 1$. The concentration of agar-agar (Roeper) was selected in the range of 0 to $1 \mathrm{~g} / \mathrm{l}$ with an increment of $0.1 \mathrm{~g} / \mathrm{l}$, carboxymethyl cellulose in the range of 1 to $12 \mathrm{~g} / 1$ with an increment of $1 \mathrm{~g} / \mathrm{l}$. The various components of the base framework of the gel (except the growth factors) were introduced into water in different proportions and mixed. The solution was sedimented, then sterilized. The resulting gel was analyzed for uniformity and viscosity, then stored in a refrigerator at $+4^{\circ} \mathrm{C}$. All samples were analyzed every day for 7 days for the formation of sediment, heterogeneities in consistency, viscosity, and color. The samples that showed the best results were mixed with each other (and then with the growth factor), after which they were also analyzed for the presence of heterogeneities in the composition, structure, color and other characteristics. As a result of the experiments, we determined the optimal range of concentrations of active substances for the essential formula of a wound healing drug, including the following active ingredients: pectin (0.5-1.0 $\mathrm{g} / \mathrm{l})$, agar-agar (0.5-1.0 g/l), alginate (0.1-0.5 g/l), chitosan $(0.1 \mathrm{~g} / \mathrm{l})$, and water-soluble cellulose derivatives (including carboxymethyl cellulose, cellosize) (10-12 g/l). Subsequently, the gel was first analyzed for the presence of specific biological activity in the culture of the human mesenchymal stem cells (hMSCs) isolated from the pulp of a human tooth. The presence of activity was measured by specific tests that determine the degree of stimulation of cells to proliferate: an increase in the level of mitochondrial activity as an indicator of energy metabolism inside the cell, the Live/Dead test.

In Stage 2, we performed technical tests of the gel composition of the medical product on hMSCs culture, and determined the optimal concentration of the growth factor, which was added to the base formula developed at Stage 1 in different concentrations from $50 \mathrm{~g} / 1$ to $1000 \mathrm{~g} / \mathrm{l}$. We used fibroblast growth factor (rhFGF-b) (Paneco, Russia). This growth factor was introduced into the gel base by mixing on a magnetic stirrer in different concentrations, after being diluted in normal saline $(\mathrm{NaCl} 0.9 \%)$. Five concentrations of rhFGF-b were studied: 50, 100, 250, 500, and $1000 \mathrm{ng} / \mathrm{ml}$. The control was a gel composition without rhFGF-b (0 ng/ml).
In the stage of technical testing, we used methods that allowed us to provide a comprehensive qualitative and quantitative analysis of the biological activity of the obtained therapeutic composition and of the characteristics of the cell culture and their activity when introduced into the culture of a medical product. We evaluated the levels of free lactate dehydrogenase (LDH) and dehydrogenase activity (MTT test), analyzed the morphological characteristics of the nuclear apparatus with nuclear staining using the Hoechst 33342 DNAbinding dye, and assessed the degree of drug influence on cell proliferation in culture, the level of mitochondrial activity, and the analysis of the active cytoskeleton. To assess the ratio of living and dead cells in the culture, the L-7007 LIVE/DEAD BacLight Bacterial Viability Kit (Invitrogen), the so-called Live/ Dead test, was used. Morphology and fluorescence staining were monitored using an LSM-510 inverted microscope (Carl Zeiss).

Cytotoxicity analysis was performed on the line of hMSCs isolated from the pulp of the teeth. Cells were plated in 96 -well plates at a density of 40,000 cells $/ \mathrm{cm}^{2}$ in DMEM/ F12 medium (1:1) supplemented with 5\% fetal calf serum and $100 \mathrm{IU} / \mathrm{ml}$ of penicillin/streptomycin and cultured under $5 \% \mathrm{CO}_{2}$ at a temperature of $37^{\circ} \mathrm{C}$. The gel composition was applied after adhesion of the culture to plastic.

In Stage 3, an experimental safety study of the developed gel composition, including growth factors, was performed on laboratory animals. Experiment was performed in accordance with the Guide for the Care and Use of Laboratory Animals (The institute of Laboratory Animal Resources, 1996).

In an experimental safety study (subchronic toxicity) on a wound model, sexually mature male rats and Wistar females of the same age (8-9 months) were used. In all animals, 2 wounds of standard size (wound radius $1.0 \mathrm{~cm}$; depth to the fascia) were modeled on the back at the same distance to the right and left of the spine and at the same distance from each other. Taking into account the weight and gender, we distributed the rats evenly into two experimental groups. Group 1 included rats we treated with a polysaccharide gel based on polymers. This polysaccharide gel included rhFGF-b. The gel was applied daily one time per day starting from day zero for 7 days; the test product was used in each animal immediately on 2 wounds. Group 2 was used as control (without treatment).

On Day 8 of the experiment, in rats under ether anesthesia, blood samples were taken from the cavity of the right ventricle for laboratory tests (clinical blood test [the content of hemoglobin, red blood cells, white blood cells and platelets] and biochemical blood test [total protein, urea, creatinine, glucose, AST and ALT]). The rats were euthanized for pathomorphological studies by closed intrathoracic transection of the great vessels and the heart, resulting in rapid exsanguination; then the absolute and relative mass of organs was determined. Organs and tissues or their parts were fixed in a $10 \%$ formalin solution. Finally, we performed a histological examination of the heart, brain, thymus, liver, kidneys, lungs, mesenteric lymph nodes, stomach, and small and large intestines in the rats of both groups with a description of the histological structure of the internal organs.

Statistical analysis was performed using statistical software package SPSS version 23.0 (SPSS Inc, Chicago, IL). The results are presented as $\mathrm{M}$ (mean) $\pm \mathrm{SD}$ (standard deviation. 
The Mann-Whitney U Test was used to compare the differences between the two independent groups. A probability value of $P<0.05$ was considered statistically significant.

\section{Results}

The analysis of the biological activity of the gel compositions modified with rhFGF-b at different concentrations $(50,100,250,500$ and $1000 \mathrm{ng} / \mathrm{ml})$ revealed their high degree of biocompatibility.

A study of cytotoxicity using different methods showed the safety of the gel composition. Thus, there was no significant increase in the level of free LDH when gel compositions were introduced in all test incubation periods (24, 48, and 72 hours), which indicates that the gel composition components did no damage to the bilayer cell membranes. The analysis of the number of apoptotic cells in the MSC culture during 24, 48, and 72 hours did not reveal a significant increase in all tested samples and in all incubation periods with gel compositions. The cells had a fibroblast-characteristic extension along the type of the leading edge and no significant increase in propidium positive stained cells. A comprehensive analysis of the morphological characteristics of the cell nuclear apparatus also did not reveal changes (condensation, degradation, chromatin fragmentation) during incubation with the modified gel compositions for up to 72 hours. This confirms the absence of a genotoxic effect. In addition, safety was confirmed by the absence of a destructive effect on the actin cytoskeleton of hMSCs when they were incubated with a gel composition after 3 days in all studied concentrations of rhFGF-b.

The next step was to determine the optimal concentration of rhFGF-b. A significant increase in the level of dehydrogenase activity was established during 3 days of cultivation at the rhFGF-b concentrations of 500 and $1000 \mathrm{ng} / \mathrm{ml}$ (Fig.1). In addition, a significant increase in the proliferative activity of hMSCs was detected at rhFGF-b concentrations of 500ng $/ \mathrm{ml}$ and $1000 \mathrm{ng} / \mathrm{ml}$. On Day 3 of cultivation, a significant increase in the number of cells in the culture was recorded. This suggests that the cells have the optimal microenvironment and maximum closed to vivo conditions (Fig.2). The absence of a significant increase in proliferative activity at lower concentrations can be attributed to the distribution of rhFGF-b in the gel composition and its diffusion to cells.

Thus, the tests performed allowed us to determine the optimal concentration of rhFGF-b at 500-1000 ng/ml, which in the polysaccharide gel composition - the basis of wound healing agents - showed the best properties. Because of the absence of significant cell changes at hFGF-b concentrations of $500 \mathrm{ng} / \mathrm{ml}$ and $1000 \mathrm{ng} / \mathrm{ml}$, given the high cost of growth factors, it was decided that a concentration of $500 \mathrm{ng} / \mathrm{ml}$ is most preferred.

The next step was an experimental study on laboratory animals, dedicated to the assessment of subtoxic toxicity of the product being developed. The results of laboratory testing showed no statistical differences in groups for various indicators. The results of gravimetric studies showed that the masses of the liver, kidneys, heart, brain, thymus, and lungs did not differ in both groups (Table 1).

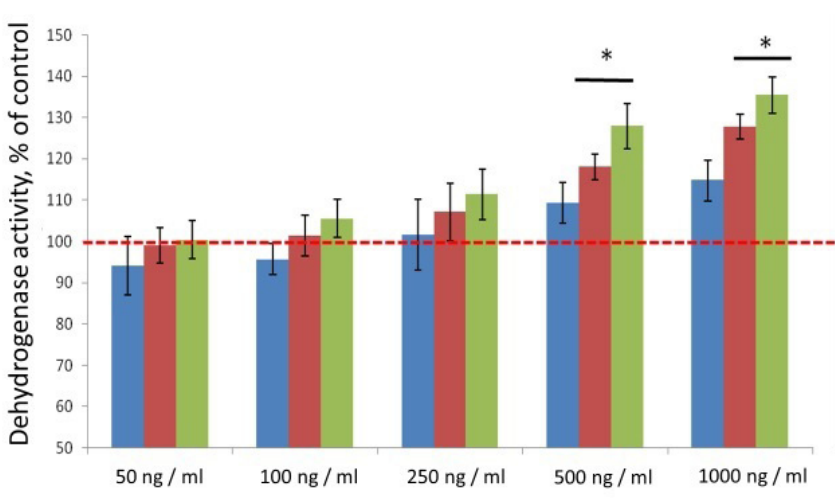

Fig. 1. Analysis of the level of dehydrogenase activity of hMSCs after incubation with a gel composition modified with rhFGF-b for 24, 48 and 72 hours.

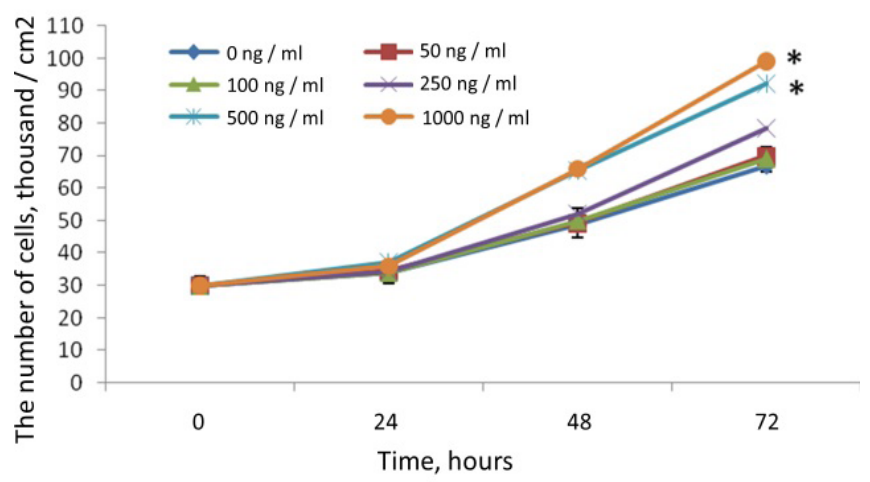

Fig. 2. Proliferation of hMSCs in the presence of a polysaccharide gel modified with rhFGF-b on Day 3 of cultivation.

Table 1. The results of laboratory testing and gravimetric studies on Day 8 of the experiment

\begin{tabular}{|l|c|c|c|}
\hline \multicolumn{1}{|c|}{ Indicator } & Group 2 & Group 1 & $P$-value \\
\hline Hemoglobin $(\mathrm{g} / \mathrm{l})$ & $137.8 \pm 4.7$ & $132.0 \pm 2.0$ & $>0.05$ \\
\hline Red blood cells $\left(\times 10^{12} / \mathrm{l}\right)$ & $6.88 \pm 0.23$ & $6.66 \pm 0.20$ & $>0.05$ \\
\hline White blood cells $\left(\times 10^{9} / \mathrm{l}\right)$ & $11.3 \pm 2.5$ & $12.8 \pm 2.5$ & $>0.05$ \\
\hline Platelets $\left(\times 10^{9} / \mathrm{l}\right)$ & $822.2 \pm 43.6$ & $800.8 \pm 47.0$ & $>0.05$ \\
\hline Total protein $(\mathrm{g} / \mathrm{l})$ & $65.8 \pm 1.3$ & $66.4 \pm 1.5$ & $>0.05$ \\
\hline Glucose $(\mathrm{mmol} / \mathrm{l})$ & $13.79 \pm 1.17$ & $14.21 \pm 1.01$ & $>0.05$ \\
\hline Urea $(\mathrm{mmol} / 1)$ & $6.6 \pm 0.3$ & $6.2 \pm 0.3$ & $>0.05$ \\
\hline Creatinine $(\mathrm{mmol} / \mathrm{l})$ & $50.4 \pm 1.9$ & $48.0 \pm 1.7$ & $>0.05$ \\
\hline AST $(\mathrm{mmol} / \mathrm{l})$ & $48.6 \pm 5.1$ & $47.6 \pm 2.8$ & $>0.05$ \\
\hline Liver weight $(\mathrm{mg} / \mathrm{g})$ & $35.3 \pm 2.2$ & $36.5 \pm 0.9$ & $>0.05$ \\
\hline Kidney weight $(\mathrm{mg} / \mathrm{g})$ & $7.3 \pm 0.3$ & $8.1 \pm 0.3$ & $>0.05$ \\
\hline Heart weight $(\mathrm{mg} / \mathrm{g})$ & $3.7 \pm 0.2$ & $4.1 \pm 0.4$ & $>0.05$ \\
\hline Brain weight $(\mathrm{mg} / \mathrm{g})$ & $6.4 \pm 0.3$ & $5.7 \pm 0.5$ & $>0.05$ \\
\hline Thymus weight $(\mathrm{mg} / \mathrm{g})$ & $1.0 \pm 0.2$ & $1.1 \pm 0.1$ & $>0.05$ \\
\hline Lung weight $(\mathrm{mg} / \mathrm{g})$ & $8.3 \pm 1.4$ & $8.5 \pm 0.6$ & $>0.05$ \\
\hline
\end{tabular}

A histological examination of the heart, brain, thymus, mesenteric lymph nodes, stomach, and small and large intestines in rats of the two groups did not reveal the presence of any pathomorphological changes. 


\section{Discussion}

Thus, on the basis of the technical tests in vitro, a gellike essential formula of a wound healing medical product was created that meets modern requirements. To improve the effect of wound healing, fibroblast growth factor was integrated into it and its optimal concentration was determined. At the same time, all used samples met safety requirements; cytotoxicity was not found. Between different research methods, a correlation of indicators was observed, that is, all indicators increase or decrease simultaneously.

The results of experimental studies have demonstrated the safety of the developed multicomponent gel composition based on natural and synthetic polymers, which includes active substances stimulating the regenerative properties of cells, namely the fibroblast growth factor. The laboratory, histological and gravimetric studies of the internal organs and wounds of the animals in the experimental and control groups did not reveal any regular pathomorphological changes indicating the presence of the general toxicological properties of the studied products. This indicates the safety in vivo of the product being developed.

The studies conducted in vitro and in vivo allow us to proceed with studies determining the effectiveness of the developed wound healing medical product on laboratory animals.

\section{Competing Interests} interests.

The authors declare that they have no competing

\section{Sources of Funding}

This work was supported by the Foundation for Assistance to Small Innovative Enterprises in Science and Technology (FASIE, Contract No. 232GRNTIS5/35963 dated 08/08/2017).

\section{References}

1. Sorg H, Tilkorn DJ, Hager S, Hauser J, Mirastschijski U. Skin wound healing: an update on the current knowledge and concepts. Eur Surg Res. 2017;58(1-2):81-94. doi: 10.1159/000454919.

2. Cañedo-Dorantes L, Cañedo-Ayala M. Skin Acute Wound Healing: A Comprehensive Review. Int J Inflam. 2019;2019:3706315. doi: 10.1155/2019/3706315.
3. Chouhan D, Dey N, Bhardwaj N, Mandal BB. Emerging and innovative approaches for wound healing and skin regeneration: Current status and advances. Biomaterials. 2019;216:119267. doi: 10.1016/j.biomaterials.2019.119267.

4. Gaspar-Pintiliescu A, Stanciuc AM, Craciunescu O. Natural composite dressings based on collagen, gelatin and plant bioactive compounds for wound healing: A review. Int J Biol Macromol. 2019;138:854-865. doi: 10.1016/j. ijbiomac.2019.07.155.

5. Oguntibeju OO. Medicinal plants and their effects on diabetic wound healing. Vet World. 2019;12(5):653-663. doi: 10.14202/vetworld.2019.653-663.

6. Deptuła M, Zieliński J, Wardowska A, Pikuła M. Wound healing complications in oncological patients: perspectives for cellular therapy. Postepy Dermatol Alergol. 2019;36(2):139146. doi: 10.5114/ada.2018.72585.

7. Silina EV, Stupin VA, Gabitov RB. [Collagen role in the mechanisms of chronic wounds healing diabetic foot syndrome]. Klinicheskaya Meditsina. 2018;96(2):106-115. doi: 10.18821/002321492018962106115. [Article in Russian]. 8. Mandla S, Davenport Huyer L, Radisic M. Review: Multimodal bioactive material approaches for wound healing. APL Bioeng. 2018;2(2):021503. doi: 10.1063/1.5026773.

9. Stupin VA, Gabitov RB, Sinelnikova TG, Silina EV. [Biological mechanisms of the chronic wound and diabetic foot healing: The rolle of collagen]. Serbian Journal of Experimental and Clinical Research. 2018;19(4):373-382. doi: 10.2478/SJECR-2018-0077.[Article in Russian].

10. Brooker JE, Camison LB, Bykowski MR, Hurley ET, Yerneni SS, Campbell PG, et al. Reconstruction of a Calvarial Wound Complicated by Infection: Comparing the Effects of Biopatterned Bone Morphogenetic Protein 2 and Vascular Endothelial Growth Factor. J Craniofac Surgery. 2019;30:260-264. doi: 10.1097/ SCS.0000000000004779.

11. Ho TC, Tsai SH, Yeh SI, Chen SL, Tung KY, Chien HY, et al. PEDF-derived peptide promotes tendon regeneration through its mitogenic effect on tendon stem/progenitor cells. Stem Cell Res Ther. 2019;10(1):2. doi: 10.1186/s13287-018-1110-z.

12. Yang R, Liu F, Wang J, Chen X, Xie J, Xiong K. Epidermal stem cells in wound healing and their clinical applications. Stem Cell Res Ther. 2019;10(1):229. doi: 10.1186/s13287019-1312-z.

13. Park JW, Hwang SR, Yoon IS. Advanced Growth Factor Delivery Systems in Wound Management and Skin Regeneration. Molecules. 2017;22(8). pii: E1259. doi: 10.3390/molecules22081259.

14. Marck RE, Gardien KLM, Vlig M, Breederveld RS, Middelkoop E. Growth factor quantification of plateletrich plasma in burn patients compared to matched healthy volunteers. Int J Mol Sci. 2019;20(2). pii: E288. doi: 10.3390/ ijms20020288. 